\title{
A new synthetic derivative of cryptotanshinone KYZ3 as STAT3 inhibitor for triple-negative breast cancer therapy
}

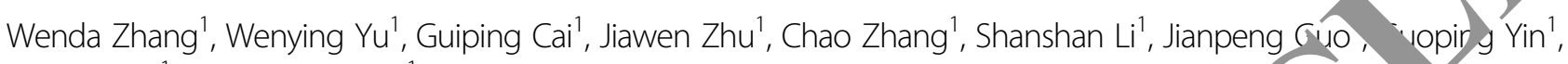 \\ Chen Chen ${ }^{1}$ and Lingyi Kong ${ }^{1}$
}

\section{Abstract}

Silencing STAT3 is confirmed as a promising therapeutic strategy for triple-negati e ast can,er (TNBC) therapy to address the issue of its poor prognosis. In this study, the natural product cryptotanshino was firstly remodeled and modified as a more effective STAT3 inhibitor by structure-based strategy. The s, thetic cerivative KYZ3 had 22-24fold increase in antitumor activity than cryptotanshinone on two TNBC cell, and little effect on normal breast epithelial MCF-10A cells. Further investigation showed that KYZ3 inhibited pe tent STAT3 phosphorylation. It also prevented the STAT3 protein nuclear translocation to regulate the exp ions ot ine target oncogenes including Bax and BCl-2. Furthermore, KYZ3 inhibited TNBC cell metastasis by decrea ing ge levels of MMP-9 which were directly regulated by activated STAT3. A STAT3 plasmid transfecting assay sugge ted that KYZ3 induced tumor cell apoptosis mainly by targeting STAT3. Finally, KYZ3 suppressed the arown. f tumors resulting from subcutaneous implantation of MDA-MB-231 cells in vivo. Taken together, KYZ3 may a pr nising cancer therapeutic agent for TNBC.

\section{Introduction}

Triple-negative breast cancers (TNBCs) are m likely to metastasize and have poor prognos without el ective drugs $^{1,2}$. Inhibiting aberrantly activat d signal transducer and activator of transcription (STAT) TN/3Cs may be a promising strategy ${ }^{3,4}$. STAT3 classifieu as an essential oncogene that regulates a master cellular events, including cancer cell $\mathrm{p}^{r}$ ratiol, apoptosis and metas-

The activation ST 3 is phosphorylated at Tyr705 residue media by gro th factor receptor tyrosine kinases and the toplasmic kinases ${ }^{9-12}$. Two phosphorylatea STAT3 pi teins form homo-dimeric-activated transcin or factor complex via reciprocal binding of

\footnotetext{
resp

${ }^{\text {SState }}$ Laboratory of Natural Medicines, Department of Natural Medicinal Chemişry, China Pharmaceutical University, 24 Tong Jia Xiang, 210009

Nanjing, China

These authors contributed equally: Wenda Zhang, Wenying Yu

Edited by A. Stephanou
} tasis $^{5-8}$.
pTyr-SH2 domains ${ }^{13-18}$. Subsequently, the complexes translocate to the nucleus and induce the target gene expression $^{19-23}$. The SH2 domain of STAT3 possesses two "hot spots", a pY705 site and a nearby pY+X site $\mathrm{e}^{24-26}$. The pY705 site is a good starting point for drug design, which mainly consists of polar residues such as Lys591, Arg609, and Glu612 responsible for binding to pTyr705 residues. While the $\mathrm{pY}+\mathrm{X}$ site is associated to the selectivity of STAT3 inhibitors. Therefore, targeting pY705 site and $\mathrm{pY}+\mathrm{X}$ site is an effective strategy for designing new STAT3 inhibitors ${ }^{27-33}$. Some of STAT3 inhibitors are on the clinical research, while there is still no STAT3 inhibitor antitumor drugs in the market ${ }^{34,35}$.

Natural products are the treasure for drug development, which have been providing novel skeletons and biological compounds to develop new drugs ${ }^{36-38}$. Nearly $60 \%$ drugs in the market are directly or indirectly derived from natural compounds ${ }^{39}$. Cryptotanshinone is a bioactive component in dried roots Salviamiltiorrhiza Bunge (Danshen) and the subject of extensive research about its 
antibacterial activity and anti-inflammation activity. While Shin Dae-Seop and coworkers reported that cryptotanshinone but not Tanshinone IIA is a STAT3 inhibitor for the potent anticancer agent by directly targeting SH2 domain in 2009 year ${ }^{40,41}$. However, its moderate potency limits it to use for cancer therapy. Therefore, structural modification of cryptotanshinone is imperative and valuable to develop more potent STAT3 inhibitors for anticancer agents.

In this study, according to the literatures and structure analysis of the binding model in silico, a new series of STAT3 inhibitors were designed by structure-based drug design strategy, and then synthesized and biologically evaluated with enhancing activity. The most potent derivative KYZ3 was elucidated as a new STAT3 inhibitor with antitumor activity against TNBCs in vitro and in vivo.

\section{Results}

\section{KYZ3 was considered as a STAT3 inhibitor and exhibited} more sensitivity to cancer cells

According to the literature and structure analysis of the binding model in silico, the saturated D ring of cryptotanshinone was essential moiety for its p-STAT3 inhibition. The methyl group on D ring was exposed to the outside of the protein surface into the water environment, which could weaken the interaction with STAT3 procenr. The A ring of cryptotanshinone increased the rigid ar $d$ just bound above the side pocket of the $\mathrm{SH}_{2}$ don which led to the poor interaction. Based these, w modified the $\mathrm{A}$ and $\mathrm{D}$ rings of cryptotanshinone shown in Fig. 1a, a new series of STAT3 i hibitors KM L-15 were designed by structure-based dr o design, and then synthesized (Fig. 1b).

Two STAT3 overactivated man INBC cell lines (MDA-MB-231 and MDA-MB 40, ere employed to test the activity of desion compounds with cryptotanshinone and BP-1-10 as nositive control by $3-(4,5-$ dimethylthiazolyl /2, 5-dipnenyltetrazoliumbro-mide (MTT) assays he $\mathrm{IC}_{50}$ values were summarized in Supplementary Ta S1. All the compounds exhibited more ant proliferative activity than cryptotanshinone and BP-1-1 14 notg them, the most potent compound KY72 inh ed he growth of MDA-MB-231 and MDAM-4 8 cells with $\mathrm{IC}_{50}$ values of $0.68 \mu \mathrm{M}$ and $0.86 \mu \mathrm{M}$, h 11 22-24-fold improvement in antiproliferation activ than cryptotanshinone (Fig. 1c). What's more, further study showed that KYZ3 was less sensitive to normal human mammary epithelial cell line MCF-10A and normal human liver cell line L02 cells (Supplementary Table S2). The data showed that our inhibitor KYZ3 had little effect on the ability of MCF-10A cells up to 5.0 $\mu \mathrm{M}$ for $48 \mathrm{~h}$. The $\mathrm{IC}_{50}$ of KYZ3 for MCF-10A cell line was $13.12 \mu \mathrm{M}$, which is 19 -fold to the $\mathrm{IC}_{50}$ of KYZ3 for MDAMB-231 cell line, $0.68 \mu \mathrm{M}$, indicating KYZ3 has certain selectivity over normal breast cell line and breast cancer cell line.

To further validate the important effect of STAT3 on KYZ3-induced cell growth inhibition, STAT3 siRNA was infected into MDA-MB-231 cells to block STAT3 and pSTAT3 (Tyr705), the expression of STAT3 and p-STAT3 (Tyr705) were both dramatically inhibited alon with the $^{2}$ decrease of cell viability. The cells that transferr were treated with KYZ3 for another $48 \mathrm{~h}$; gell viabilit further decreased by KYZ3 (Fig. 1e), beca of its further ablation of p-STAT3. All these data indica that a new derivative of cryptotanshinone, YZ3, was capable of antitumor activity due to its døwn rulation of p-STAT3.

KYZ3 featured much bet actr. than the parent molecule cryptotanshinone. To vestigate the mechanism of the improved titumo activity, firstly, the potential binding mode the STAT3 protein was predicted by dockin experinnents. As Fig. 1d illustrates, KYZ3 bound the STAT3 SH2 domain by forming an essen bydrogen bonding interaction with the Arg60 cidue of STAT3 protein, which was similar to the bin lirg y rodes of cryptotanshinone. In addition, the 2-methvl group of KYZ3 fitted deeply into the P . 705 pocket compared to the 3-methyl group of crypt anshinone exposure to the water environment. thermore, the flexible side chain of KYZ3 on B ring could tightly bind to the side pocket. Together, these data provided a rationale explaining the markedly increased activity of KYZ3 as a STAT3 inhibitor.

\section{KYZ3 inhibited the phosphorylation nuclear translocation of STAT3, and regulated the expression of STAT3 target oncogenes \\ The effect of KYZ3 on STAT3 phosphorylation was} investigated by the western blot analysis. MDA-MB-231 and MDA-MB-468 cells were incubated with KYZ3 and the levels of p-STAT3 were then examined. KYZ3 suppressed STAT3 phosphorylation at Tyr705 residue in a dose-dependent manner, but had no effects on the total STAT3 levels in both cell lines (Fig. 2a). Nuclear translocation of STAT3 plays a critical role in STAT3:STAT3/ DNA complex formation and its target gene expression. The p-STAT3 protein was retained in both cytoplasm and nucleus in MDA-MB-231 cells treated without KYZ3. While treatment with KYZ3 led to a significant decrease of the p-STAT3 level in nucleus and cytoplasm. The fluorescence intensity is weaker in the nucleus than cytoplasm in MDA-MB-468 cells; treatment with KYZ3 led to a decrease of the p-STAT3 level both in the nucleus and cytoplasm, but mainly in the cytoplasm (Fig. 2b). To gain more insights into the mechanistic effects of KYZ3 on the STAT3 pathway, its effect on the expression of STAT3-targeted genes was examined. KYZ3 inhibited the expressions of Bcl-2, and increased the levels of Bax in a 


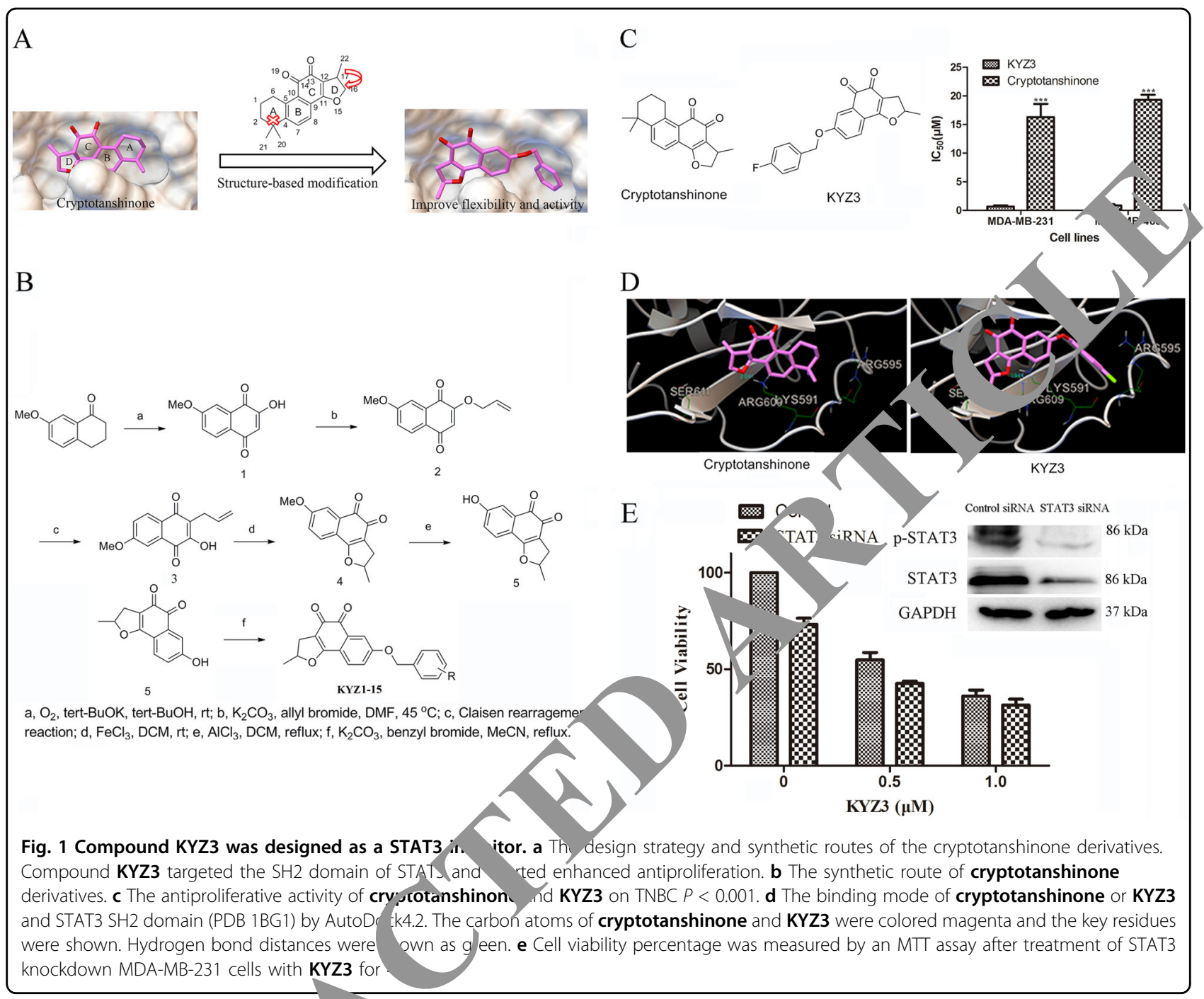

dose-dependent ma or both MDA-MB-231 and MDA-MB-468 ce ${ }^{1 / 2}\left(\mathrm{Fi}_{c}{ }^{\mathrm{c}} \mathrm{c}, \mathrm{a}\right)$.

KYZ3 inhibitec car cell migration and the expression of MMP-9 ard MMP-2 $p_{\text {, }}$,teins

Migra n s directly or indirectly responsible for the mainity o ancer deaths. Antimetastasis treatment is thus $\mathrm{e}$ e key, 0 cure cancer ${ }^{42}$. To investigate the inhibitory e. L YZ3 on tumor migration, a wound healing assa ras performed. In the presence of KYZ3 at $0.25 \mu \mathrm{M}$, the migration of MDA-MB-231 cells was effectively suppressed, and was almost inhibited at $0.5 \mu \mathrm{M}$ KYZ3 (Fig. 3a). Next, the trans-well invasion assay was further performed; a significant reduction in invasive ability at $0.25 \mu \mathrm{M}$ of KYZ3 and an almost cessation of cancer migration at $0.5 \mu \mathrm{M}$ KYZ3 were observed (Fig. 3b). Matrix metalloproteinase (MMP) enzyme MMP-9 and MMP-2 control the epithelial-mesenchymal transition, and their expression levels are directly regulated by the activated STAT3 ${ }^{3,4,43}$. The effect of KYZ3 on MMP-9 and MMP-2 expression levels was investigated. A dosedependent decrease of MMP-9 and MMP-2 was induced by KYZ3 (Fig. 3c, d). These data suggested that KYZ3 inhibited cancer cell migration by decreasing the levels of MMP-9 and MMP-2.

Previous experiments indicated that KYZ3 inhibited the phosphorylation of STAT3. To investigate whether the effect of KYZ3 could be recovered partially with further overexpression of p-STAT3 in MDA-MB-231 cells, we transfected the MDA-MB-231 cells with further STAT3; thus the p-STAT3 level was also increased. As a result, KYZ3 could inhibit p-STAT3 level but did not affect the total STAT3 level. The inhibition of the p-STAT3 level of the transfected cells was recovered a little bit upon treatment with $2 \mu \mathrm{M}$ of KYZ3 (Fig. 3e) ${ }^{* * *} p<0.01$, $* p<0.001$. 


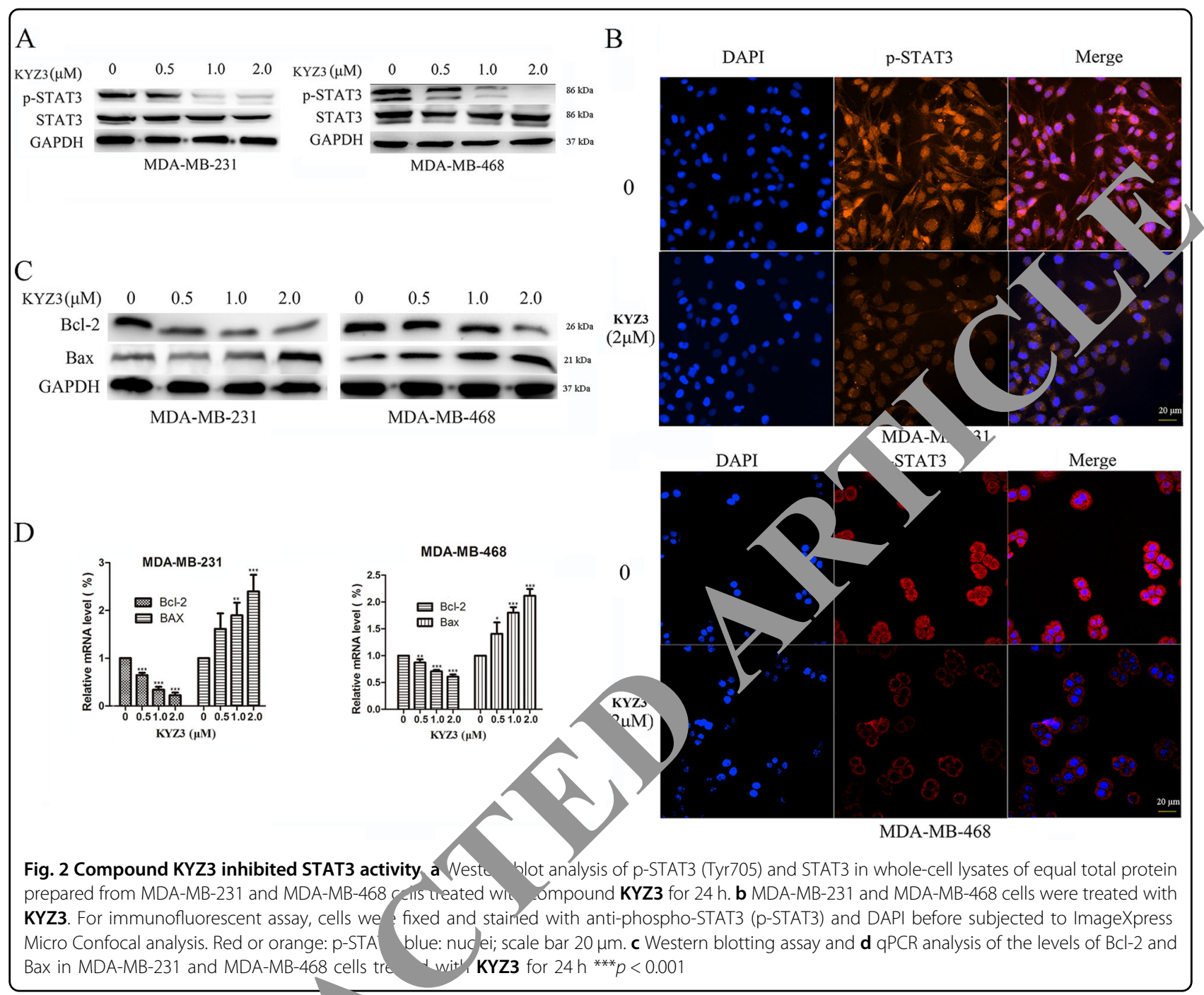

KYZ3 induced the RC ren mation out did not inhibit the phosphorylation $c$, the strearn kinases

The activatio, of STA, phosphorylated at Tyr705 residue mav se $m$ iated by the upstream kinases. The effect of $\mathrm{NZ3}$ on 1 Src and p-Erk protein levels was evaluan by the western blot analysis. Data showed that KY72 hac tle effect on p-Src, and p-Erk expressions, adic ing tha the inhibition of KYZ3 on p-STAT3 was n Eran with inhibiting p-Src and p-Erk (Fig. 4a).

T. moderate level of reactive oxygen species (ROS) is sufficient for cancer cells to show active metabolism in response to oncogenic signal. In general, cancer cells take advantage of this moderate oxidative stress for proliferation, metastasis, and angiogenesis and so on. Nevertheless, high levels of ROS irreversibly damage DNA and lipids and ultimately cause cancer cell apoptosis ${ }^{44}$. The effect of KYZ3 on the ROS levels in tumor cells was evaluated. The levels of ROS showed 1.2-, 1.3-, and 1.6-fold increases in
MDA-MB-231 cells (Fig. 4b) and 1.1-, 1.4-, and 1.9-fold increases in MDA-MB-468 cells at $0.5,1.0$, and $2.0 \mu \mathrm{M}$ of KYZ3 (Fig. 4c), indicating that KYZ3 induced ROS accumulation which may partially contribute to its selective inhibition on cancer cell proliferation and metastasis.

\section{KYZ3 induced breast cancer cell apoptosis but did not} affect MCF-10A breast epithelial cells

The mechanism of cancer cell apoptosis induced by KYZ3 was investigated. The effect of KYZ3 on tumor cell apoptosis was analyzed by flow cytometry. Annexin VAPC/7-AAD staining was carried out and determined using flow cytometry. KYZ3 dose-dependently induced the apoptosis of MDA-MB-231 and MDA-MB-468 cells. The induced apoptosis rates at $0,1.0,2.0$, and $4.0 \mu \mathrm{M}$ were 4.6, 33.9, 64.9, and 67.5\% (Fig. 5a), and 2.9, 17.5, 26.2, and 47.1\% (Fig. 5b), respectively. However, there was little apoptosis induced by $\mathrm{KYZ3}$ at $4.0 \mu \mathrm{M}$ in MCF-10A cells 


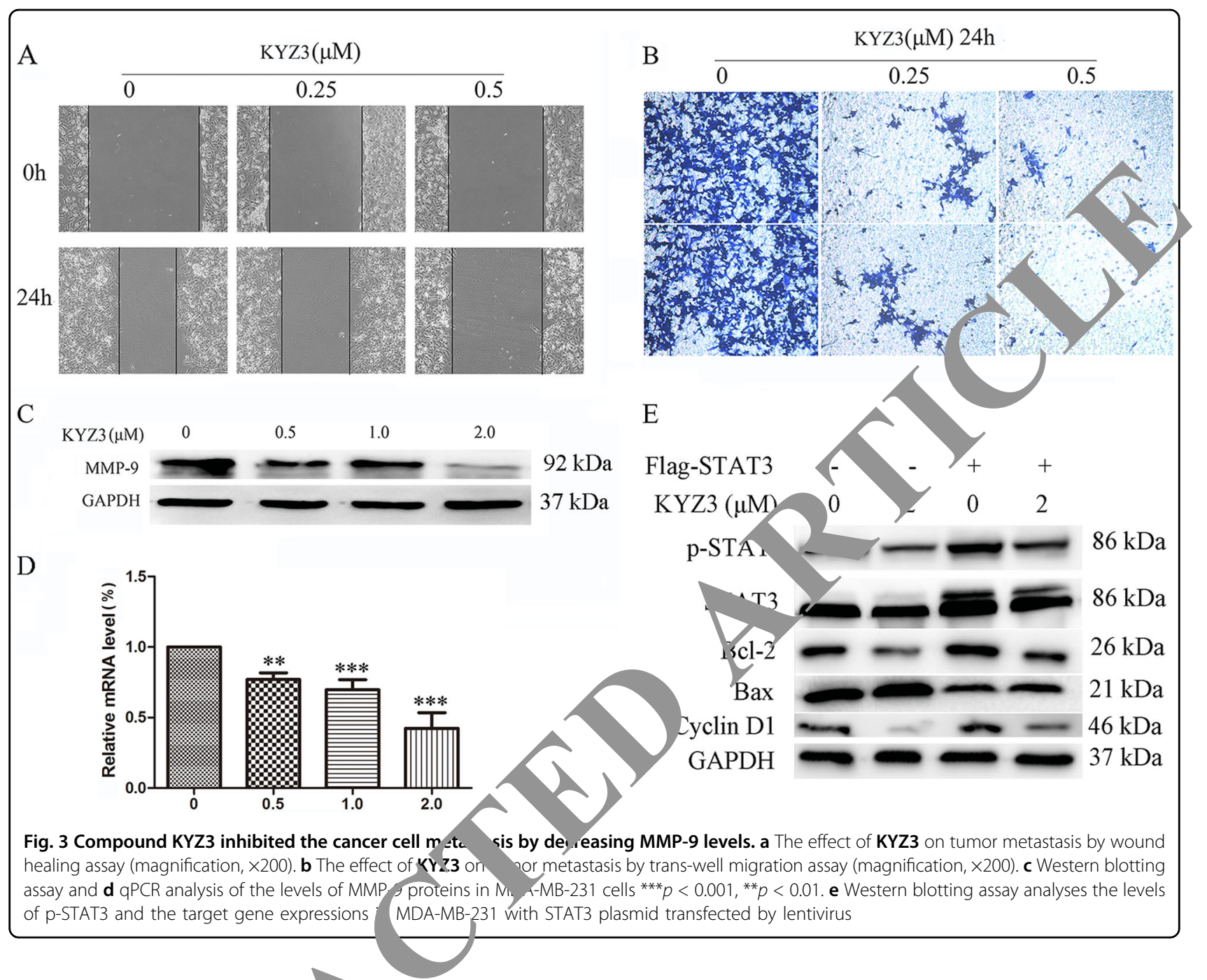

(Fig. 5c), further indic that KYZ3 was a selective antitumor agent $p$ ribl throagh Bax/Bcl-2-related caspase-dependen ap tosis pathway by inhibiting STAT3 activity. Uestern b $\subset$ analysis revealed that KYZ3 increased the cle. oe levels of PARP and Caspase 3 proteins in MDA-1 B-231 and MDA-MB-468 cells. Taken ef er, all these results further demonstrated that ${ }^{+}$YZs du ced cell apoptosis by targeting STAT3.

\section{$h$. Ited antitumor activity in vivo}

To valuate the in vivo anticancer activity of KYZ3, $\mathrm{BALB} / \mathrm{c}$ nude mice were inoculated subcutaneously with human breast cancer cells MDA-MB-231. Treatment with $15 \mathrm{mg} / \mathrm{kg}$ of KYZ3 reduced the volumes of the implanted human breast tumor, while treatment with $30 \mathrm{mg} / \mathrm{kg}$ of KYZ3 further inhibited the growth of the implanted tumors (Fig. 6a). Importantly, the weight of tumor tissues from mice treated with KYZ3 was significantly reduced by 36.2 or $59.4 \%$, and there was no significant body weight loss (Fig. 6b). Immunohistochemistry analysis of lysates from the tumor tissues treated with KYZ3 showed a suppression of Ki67 compared with the control tumors (Fig. 6c) upon H\&E staining. We stained Ki67 with H\&E and quantified the Ki67 expression across a much larger field area. The results (Fig. 6c) showed that the inhibition of Ki67 expression in T1 was about $47 \%$ and in T2 was about $65 \%$. We have investigated the levels of p-STAT3 in the control and treated tumors by immunohistochemistry. As Fig. 6d shows, KYZ3 inhibited the levels of p-STAT3 in vivo. These results showed that KYZ3 had potent antitumor activity against the growth of implanted human breast tumors with little toxicity.

\section{Discussion}

Triple-negative breast cancer is the leading cause of cancer-related morbidity and mortality in women. It is more likely to metastasize and have poor prognosis without special drugs ${ }^{1}$. Therefore, there is a paucity of 


\section{A}

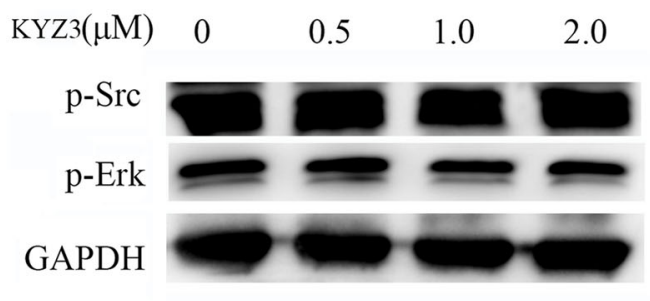

MDA-MB-231

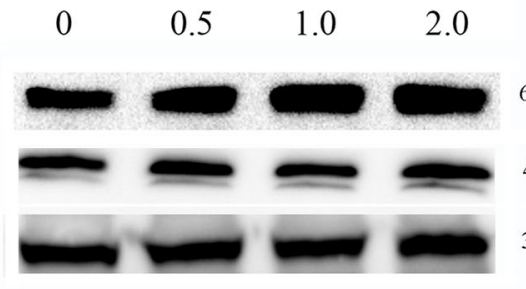

$60 \mathrm{kDa}$

MDA-MB-468

B
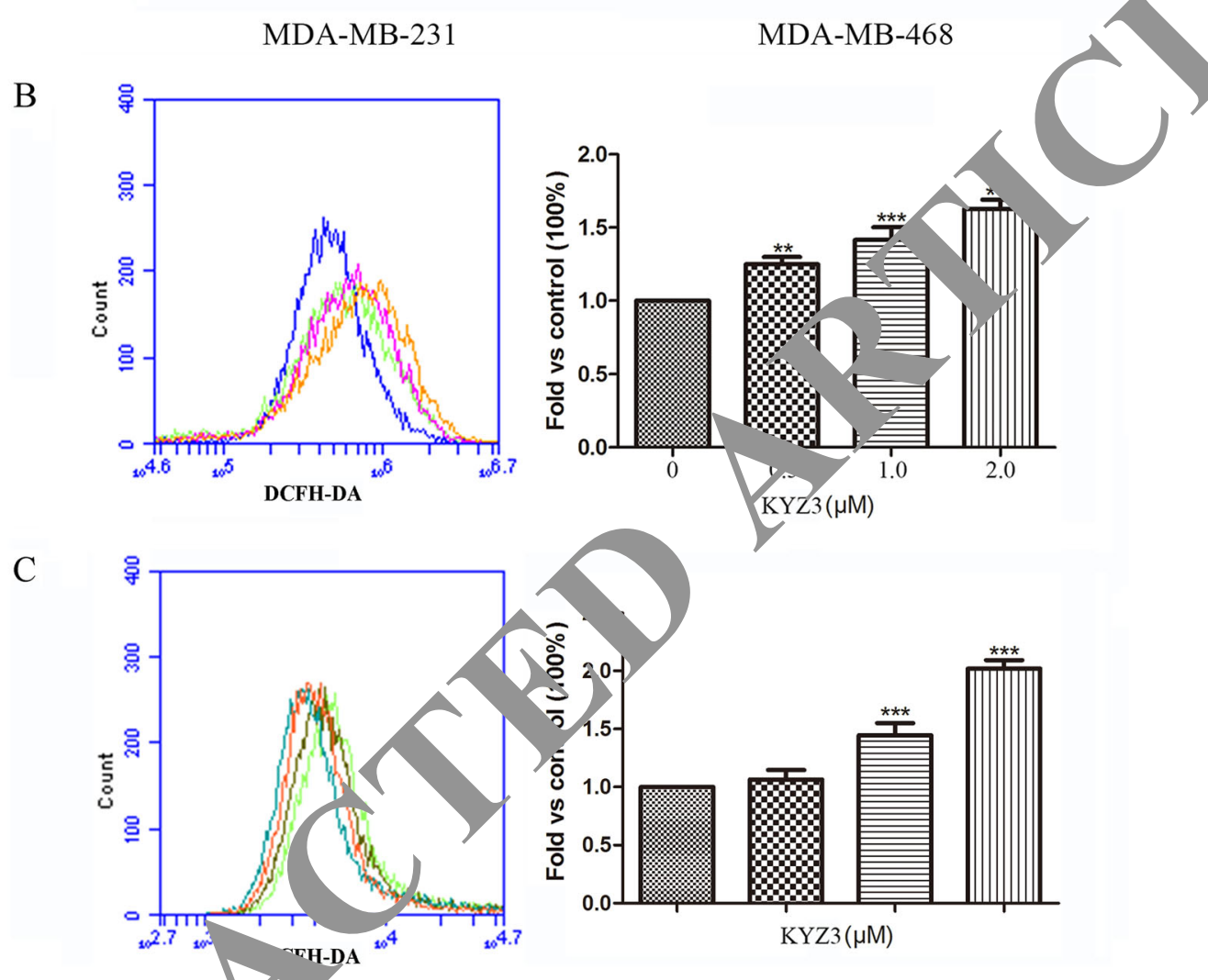

$\operatorname{KYZ3}(\mu \mathrm{M})$

Fig. 4 Compound KYZ3 ind - ROS -eneration without affecting the upstream kinases of STAT3. a Western blot analysis of p-Src and p-Erk levels in whole-cell lysates of ea, total stein prepared from MDA-MB-231 and MDA-MB-468 cells treated with compound KYZ3 for $24 \mathrm{~h}$. MDAMB-231 (b) and MDA-MBB- con incubated with $\mathbf{K Y Z 3}$ at different concentrations for $24 \mathrm{~h}$, and then cells were collected and stained by DCFH-DA and subje <tea to in cytometry. ${ }^{* * *} p<0.001,{ }^{* *} p<0.01$

effectiv ea neits for managing patients with metastatic $\mathrm{TN}^{\mathrm{DP} \mathrm{S}^{2}}$. $\mathrm{L}$ biting aberrantly activated signal transducer nd a civator of transcription (STAT) 3 in TNBC may be a

$\mathrm{S} 1$ T3 is classified as an essential oncogene that regulates a master of the cellular events, including cancer cell proliferation, apoptosis, and metastasis ${ }^{5-8}$. The $\mathrm{SH} 2$ domain of STAT3 plays a critical role in the recruitment of STAT3 activation and the formation of STAT3 homodimers along with the formation of STAT3:STAT3/ DNA complex ${ }^{24,25}$. Thus, designing STAT3 inhibitor targeting $\mathrm{SH} 2$ domain is an effective strategy to silence STAT3 activity $^{27-33}$.
Natural products provide a varied and complex template structure for drug development. Cryptotanshinone is a bioactive component present in the dried roots of Salviamiltiorrhiza Bunge (Danshen), and was reported, in the year 2009, as a STAT3 inhibitor for the potent anticancer agent by directly targeting the $\mathrm{SH} 2$ domain $^{39,45}$. However, its moderate potency limits its use for cancer therapy and its modification has not been reported till now.

This is the first study to report on the design, synthesis, and biological evaluation of cryptotanshinone analogs as new STAT3 inhibitors. According to the structure-based drug design strategy, a series of new STAT3 inhibitors 


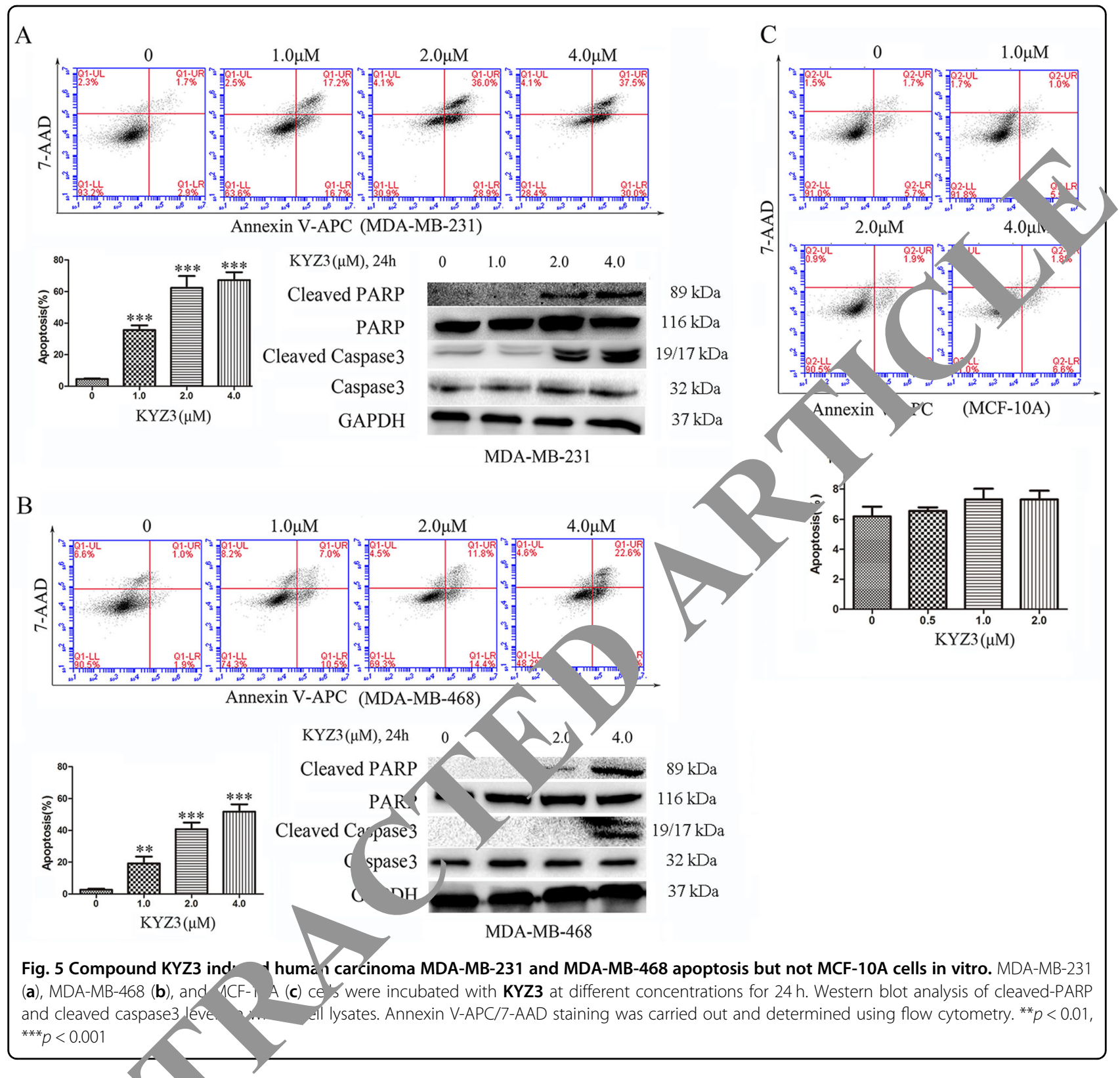

were ob in Epecially, the derivative KYZ3 exhibited 22-11-fola ncrease in antitumor activity against two + pic: TNBC cell lines with less sensitivity to MCF-10A a an $L 02$ cells. Docking studies discovered KYZ3 targ y the STAT3 SH2 domain. Targeting this domain not only could inhibit STAT3 phosphorylation but also could suppress the STAT3:STAT3/DNA-binding complex forming which regulates downstream oncogene expression. Subsequently, the investigation of antitumor mechanism showed that KYZ3 inhibited persistent and IL-6-induced STAT3 phosphorylation, STAT3 nuclear translocation and regulated the expression of STAT3 target genes such as Bax and Bcl-2. Bax/Bcl-2 are apoptosis genes; the increasing ratio of them induced by KYZ3 activated the caspase-dependent apoptosis pathway of TNBCs. More importantly, KYZ3 inhibited cancer cell metastasis by decreasing the expression of MMP-9, which were directly regulated by activating STAT3, suggesting that KYZ3 may be a promising antimetastasis agent. Additionally, the ROS levels of cancer cells were increased by KYZ3, which further induced the tumor cell apoptosis and increased the antiproliferative selectivity against cancer cells. Furthermore, KYZ3 in vivo induced significant antitumor responses, and exhibited no observed toxicity. In summary, KYZ3 was confirmed as a new STAT3 inhibitor and exhibited marked antitumor activity, 


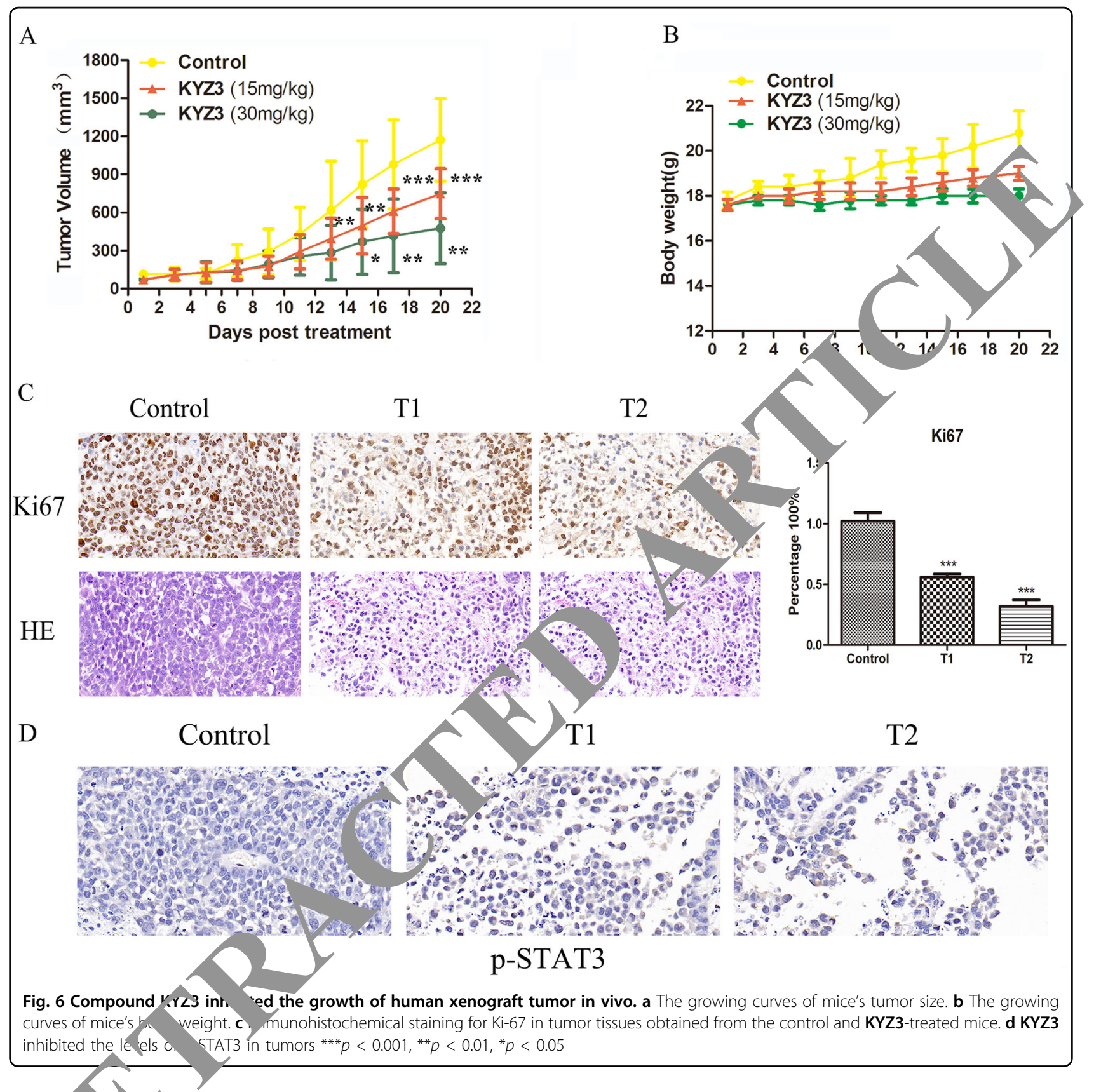

whirh is rtby of further investigation in order to evel offec ave STAT3 inhibitors for TNBC.

\section{Ma. ials and methods \\ Chemistry}

The design and synthetic strategy for compounds KYZ1-16 are shown in Fig. 1. Positive control cryptotanshinone and BP-1-102 was purchased from commercial suppliers. All of the cryptotanshinone derivatives were confirmed by ${ }^{1} \mathrm{H}$ NMR, IR, HRMS (ESI) spectra, and ${ }^{13} \mathrm{C}$ NMR spectrum, and are shown in Supplementary Materials and Methods.

\section{Docking}

Computational docking program AutoDock4.2 was used to dock KYZ3 to predict the binding mode. Briefly, compound KYZ3 was docked using the Lamarckian Genetic Algorithm. The ligand and macromolecule were prepared using the Schrödinger software. Then, AutoGrid maps were prepared for all atom types. After 10 million energy evaluations were completed, the root-mean-square deviation threshold was set as $1.5 \AA$ and all the resulting conformations of the ligands in the binding pocket of the macromolecule were clustered. 
Maintenance of cell lines culture and cell viability assays

All cell lines were purchased from Cell Bank of Shanghai Institute of Biochemistry and Cell Biology, Chinese Academy of Sciences (Shanghai, China). MtrDNA sequence analysis was done by the Cell Bank of Shanghai Institute of Biochemistry and Cell Biology, Chinese Academy of Sciences, Key GEN Bio TECH Co., Ltd., and Wuhan Gene Create Biological Engineering Co., Ltd. Human breast carcinoma cells MDA-MB-231 and MDA-MB-468, breast epithelial cells MCF-10A and human normal liver cells L02 were maintained in RPMI1640 medium. All cells were supplemented with $12 \%$ fetal bovine serum containing $50 \mu \mathrm{g} / \mathrm{mL}$ penicillin and $50 \mu \mathrm{g} / \mathrm{mL}$ streptomycin. Cells were grown to $80 \%$ confluency in a tissue culture flask at $37^{\circ} \mathrm{C}$ in a humidified atmosphere containing $5 \% \mathrm{CO}_{2}$, and then were trypsinized and splitted.

Cells were seeded in 96-well plates at a density of $3000-6000$ cells per well. The cells were incubated at $37^{\circ}$ $\mathrm{C}$ overnight. After medium removal, different concentrations of test compounds were added in triplicate to the plates in $200 \mu \mathrm{L}$ fresh mediums; the plates were incubated at $37^{\circ} \mathrm{C}$ for $72 \mathrm{~h}$. Then $20 \mu \mathrm{L}$ MTT was added to evaluate cell viability. The absorbance was read by an ELISA reader (SpectraMax Plus384, Molecular Devices, Sunnyvale, CA) at a test wavelength of $570 \mathrm{~nm}$ ara a reference wavelength of $630 \mathrm{~nm}$. Cell viability was $1 \mathrm{cl}$. lated by the following formula:

$$
\% \text { Cell viability }=(A t / A s) \times 100 \% .
$$

At and As denoted the absorbance of the test subs,ances and solvent control, respectively.

\section{Western blot analysis}

Cells were incubated with virre. -oncentrations of KYZ3 for $24 \mathrm{~h}$. Harvest: fter irypsinisation, cells were treated with $1 \times \mathrm{RIP} /$ 'vsis huffer $(50 \mathrm{mM}$ Tris- $\mathrm{HCl}, \mathrm{pH}$ 7.4, $150 \mathrm{mM} \mathrm{NaC}, 0.2$ deoxycholic acid, 1\% NP-40,1 mM EDTA ar. proteast hhibitors) (Amresco, Solon, USA) to extract th otal proteins. An aliquot of proteins from the cotal cell ly ates (30-60 $\mu \mathrm{g} / \mathrm{lane})$ was separated by sou $\square$ dod ${ }^{2}$ cyl sulfate (10\%) polyacrylamide gel electmopho is SDS-PAGE, BioRad Laboratories, Herles, CA), vet-transferred to NC membrane (BioRad L. Mawns, Hercules, CA) and blotted with primary antik ties specific for STAT3, p-STAT3, p-Src, P-Erk, Cleaved-PARP, PARP, Cleaved Caspase3, Caspase3, Bcl-2, Bax, MMP-9, and GAPDH. Bound immuno-complexes were detected using Chemi DOC ${ }^{\mathrm{m}} \mathrm{XRS}+$ system (BioRad Laboratories, Hercules, CA).

\section{Immunofluorescence staining assays}

Cells $\left(1 \times 10^{4} /\right.$ well $)$ were cultured on 96 -well glass culture plate (Corning, USA). After incubation with different concentrations of KYZ3, cells were fixed with $4 \%$ paraformaldehyde for $15 \mathrm{~min}$, and permeabilized with $0.5 \%$ Triton X-100 for $15 \mathrm{~min}$. Next, the cells were blocked with $5 \%$ BSA for $1 \mathrm{~h}$ and incubated with the primary antibody overnight at $4{ }^{\circ} \mathrm{C}$. Alexa-conjugated secondary antibodies (Alexa Fluor 594 goat anti-rabbit IgG Alexa Fluor 488 goat anti-rabbit IgG, Cell Signaling Te nnology, MA, USA) were applied and incubated at rou temperature for $1 \mathrm{~h}$. Cell nucleus was stained $\mathrm{w}_{\mathrm{1}} 4$, 6-diamino-2-phenyl indole (DAPI, Celi rnaling Technology, MA, USA) for $10 \mathrm{~min}$. Fin iny, the ils images were analyzed by ImageXpress ${ }^{\circledR}$ Iicro Conf, cal (Molecular Devices, USA).

\section{Quantitative real-time RT-PCR}

Levels of mRNA ex re on were analyzed with the RTPCR assay, with total RN olated from cells using an EASY spin Plus tro re/cell $\ll$ NA extraction kit (Aidlab Biotechnologie 0 RNA was quantified by measuring absorption $260 \mathrm{~nm}$ and $1 \mu \mathrm{g}$ of RNA was reverse transcribe CDN/ using the Transcriptor First Strand cDNA Syntbesis Kit (Roche Diagnostics, Basel, Switzerland). Ther nal cycling conditions included $95^{\circ} \mathrm{C}$ initial at. uration for $5 \mathrm{~min}$, followed by 40 cycles of denaturat $\mathrm{n}\left(10 \mathrm{~s}\right.$ at $\left.95^{\circ} \mathrm{C}\right)$, annealing $\left(15 \mathrm{~s}\right.$ at $\left.60^{\circ} \mathrm{C}\right)$ and ension $\left(15 \mathrm{~s}\right.$ at $72^{\circ} \mathrm{C}$ with a single fluorescence measulement), a melt curve program $\left(60-95^{\circ} \mathrm{C}\right.$ with a $0.11^{\circ} \mathrm{C} / \mathrm{s}$ heat increase and continuous fluorescence measurement) and a cooling step to $40^{\circ} \mathrm{C}$. The $\Delta$ cycle threshold method was used for the calculation of relative differences in mRNA abundance with a Light Cycler 480 (Roche Molecular Biochemicals, Mannheim, Germany). The data were normalized to the expression of GAPDH. The results were expressed as fold-changes. The RT-PCR primers that were used in this study are listed in the supporting information.

\section{Wound healing assays}

Cells were seeded at $6 \times 10^{5}$ cells per well into six-well plate and allowed to grow overnight. Wounds were made by scratching the cells with pipette tips $(1-10 \mu \mathrm{L})$. Cells were treated with $\mathbf{K Y Z 3}$ and allowed to migrate into the scratched area for $24 \mathrm{~h}$. The migration of cells was visualized at $\times 10$ magnification using a Leica Microscope at time $0 \mathrm{~h}$ (immediately before the drug was added) and $24 \mathrm{~h}$.

\section{Trans-well invasive assays}

Cells were seeded at $3 \times 10^{4}$ cells per well and allowed to grow overnight. The following day, the cells were serumstarved. Cells were treated with KYZ3 and allowed to migrate into the scratched area for $24 \mathrm{~h}$ after being fixed in $4 \%$ paraformaldehyde for $10 \mathrm{~min}$. Crystal violet solution (Sigma, St. Louis, MO, USA) was used to stain the 
colonies for $4 \mathrm{~h}$. The migration of cells was visualized at $\times 10$ magnification using a Leica Microscope.

\section{Apoptotic assay}

Cells at a density of $2.5 \times 10^{5}$ per well were cultured in regular growth medium in six-well plates for $24 \mathrm{~h}$ and treated in duplicate with different concentrations of KYZ3 for $24 \mathrm{~h}$. The cells were harvested, washed and stained with $5 \mu \mathrm{L}$ Annexin V-APC and $5 \mu \mathrm{L} 7$-AAD at room temperature for $15 \mathrm{~min}$. Cells were then analyzed by flow cytometry $(488 \mathrm{~nm}$ excitation and $600 \mathrm{~nm}$ emission filters) using BD FACSCalibur flow cytometer (Becton \& Dickinson Company, Franklin Lakes, NJ).

\section{Detection of ROS}

Cells were seeded at a density of $3 \times 10^{5}$ per well of sixwell plates. The cells were treated with various concentrations of KYZ3 for $24 \mathrm{~h}$. The growth media was replaced with serum-free medium containing a final concentration of $10 \mu \mathrm{M}$ DCFH-DA probe. After incubation for $20 \mathrm{~min}$ at $37^{\circ} \mathrm{C}$, cells were washed with serumfree medium twice, digested by trypsin and resuspended in the prewarmed PBS buffer. The samples were then subjected to a flow cytometry assay using BD FACSCalibur flow cytometer (Becton \& Dickinson Company, Franklin Lakes, NJ).

\section{In vivo studies}

Four-week-old, $20 \mathrm{~g}$, female BALB/c nude $(16-1)$ g) were injected with human breast tumor MDA 'B-231 $\left(3 \times 10^{6}\right.$ cells in a volume of $\left.0.2 \mathrm{~mL}\right)$ int $\sigma$ the subcut , neous tissue of the mice. Three days at or the fumor cell inoculation, the mice were randoml orted into three groups with six mice per group The tumor-bearing mice were either given an i.p. injection $10, \mathrm{~kg}$ or $30 \mathrm{mg} / \mathrm{kg}$ of compound KYZ3. The tment was initiated when the tumor burden of $\mathrm{n} r$ ched around $80 \mathrm{~mm}^{3}$. The tumor size was $m$ usur three times a week and calculated by the fo ula: lel,$h \times$ width $^{2} / 2$, and the body weight was masu. and recorded. On the 22nd day, all mice we killed, an the tumor was segregated, and weighe Th letels of p-STAT3 in tumor tissues were anal-ned $\mathrm{b}$, he western blot assay.

The exper mental mice were cared for and handled S. ry an ording to the recommendations of the Animal Eth Committee of China Pharmaceutical University, and the National Institutes of Health (NIH) standard guidelines for the Care and Use of Laboratory Animals. All experimental protocols were approved by the Animal Ethics Committee of China Pharmaceutical University.

\section{Statistical analysis}

Statistical analysis was performed with ANOVA by using Graph Pad Prism version 5.0 (Graph Pad Software,
San Diego, CA, USA). The results were given as the mean \pm SD. $p<0.05$ was considered significant.

\section{Acknowledgements}

This research work was supported by National Natural Science Foundation of China (Grant No. 81673298 and No. 81402791), Natural Science Foundation of the Jiangsu Higher Education Institutions of China for Excellent Youn Talents (Grant No. BK20180077), Jiangsu Shuangchuang Talents Group, Pr Jgram for Changjiang Scholars, Innovation Program of Jiangsu Province (I V17 0731) and Innovative Research Team in University (IRT_15R63), project fur. Priority Academic Program Development of Jiangsu Higher Educatio Institutions (PAPD), the 111 Project from Ministry of E tation of China and the State Administration of Foreign Export Affairs of Chis Vo. B18 j6).

\section{Authors' contributions}

W.Z. and W.Y. carried out the experimental $W$ experiments L.K. provided oversight.

experiments and wrote the manuscript.

\section{Conflict of interest}

The authors declare that the have conflict of interest.

Publisher's note

Springer Nature remains utral with regard to jurisdictional claims in published $m$ and instity onal affiliations.

Supplementaiv infor nation accompanies this paper at (https://doi.org/ 10.1038/s41419-018-1139-z).

Receiv : 5 July 2018 Revised: 3 October 2018 Accepted: 4 October 2018 blis ed online: 27 October 2018

\section{References}

1. Cheng, Y. et al. XPO1 (CRM1) inhibition represses STAT3 activation to drive a survivin-dependent oncogenic switch in triple-negative breast cancer. Mol. Cancer Ther. 13, 675-686 (2014).

2. Lee, H. J. et al. Oral administration of penta-O-galloyl-beta-D-glucose suppresses triple-negative breast cancer xenograft growth and metastasis in strong association with JAK1-STAT3 inhibition. Carcinogenesis 32, 804-811 (2011).

3. Liu, C. Y. et al. Obatoclax analog SC-2001 inhibits STAT3 phosphorylation through enhancing SHP-1 expression and induces apoptosis in human breast cancer cells. Breast Cancer Res Tr. 146, 71-84 (2014).

4. Shields, B. J. et al. TCPTP regulates SFK and STAT3 signaling and is lost in triplenegative breast cancers. Mol. Cell Biol. 33, 557-570 (2013).

5. Schust, J., Sperl, B., Hollis, A., Mayer, T. U. \& Berg, T. Stattic: a small-molecule inhibitor of STAT3 activation and dimerization. Chem. Biol. 13, 1235-1242 (2006).

6. Song, H., Wang, R., Wang, S. \& Lin, J. A low-molecular-weight compound discovered through virtual database screening inhibits STAT3 function in breast cancer cells. Proc. Natl. Acad. Sci. USA 102, 4700-4705 (2005).

7. Banerjee, K. \& Resat, H. Constitutive activation of STAT3 in breast cancer cells: a review. Int. J. Cancer 138, 2570-2578 (2016).

8. Kortylewski, M., Jove, R. \& Yu, H. Targeting STAT3 affects melanoma on multiple fronts. Cancer Metastas-. Rev. 24, 315-327 (2005).

9. Fuh, B. et al. LLL-3 inhibits STAT3 activity, suppresses glioblastoma cell growth and prolongs survival in a mouse glioblastoma model. Br. J. Cancer 100, 106-112 (2009).

10. Chen, H. et al. Fragment-based drug design and identification of HJCO123, a novel orally bioavailable STAT3 inhibitor for cancer therapy. Eur. J. Med. Chem. 62, 498-507 (2013).

11. Chen, H. et al. Discovery of alkylamino tethered Niclosamide derivatives as potent and orally bioavailable anticancer agents. ACS Med. Chem. Lett. 4, 180-185 (2013).

12. Chen, $\mathrm{H}$. et al. Discovery of potent anticancer agent HJC0416, an orally bioavailable small molecule inhibitor of signal transducer and activator of transcription 3 (STAT3). Eur. J. Med. Chem. 84, 195-203 (2014). 
13. Daka, P. et al. Design, synthesis and evaluation of $\mathrm{XZH}-5$ analogues as STAT3 inhibitors. Bioorg. Med. Chem. 23, 1348-1355 (2015).

14. Fletcher, S. et al. Disruption of transcriptionally active STAT3 dimers with nonphosphorylated, salicylic acid-based small molecules: potent in vitro and tumor cell activities. Chembiochem 10, 1959-1964 (2009).

15. Haftchenary, S. et al. Potent targeting of the STAT3 protein in brain cancer stem cells: a promising route for treating glioblastoma. ACS Med. Chem. Lett. $\mathbf{4}$, 1102-1107 (2013).

16. Hao, W. et al. Discovery of the catechol tructural moiety as a STAT3 SH2 domain inhibitor by virtual screening. Bioorg. Med. Chem. Lett. 18, 4988-4992 (2008).

17. $\mathrm{Xu}, \mathrm{J}$. et al. Inhibition of the signal transducerandactivator of transcription-3 (STAT3) signaling pathway by 4-oxo-1-phenyl-1,4-dihydroquinoline-3-carboxylic acid esters. J. Med. Chem. 51, 4115-4121 (2008).

18. Siddiquee, K. A. et al. An oxazole-based small-molecule STAT3 inhibitor modulates STAT3 stability and processing and induces antitumor cell effects. ACS ChemBiol. 12, 787-798 (2007).

19. Ge, J., Wu, H. \& Yao, S. Q. An unnatural amino acid that mimics phosphotyrosine. Chem. Commun. 46, 2980-2982 (2010).

20. Leung, K. H. et al. Discovery of a small-molecule inhibitor of STAT3 by ligandbased pharmacophore screening. Methods 71, 38-43 (2015).

21. $\mathrm{Li}, \mathrm{H}$. et al. Fragment-based drug design and drug repositioning using multiple ligand simultaneous docking (MLSD): identifying celecoxib and template compounds as novel inhibitors of signal transducer and activator of transcription 3 (STAT3). J. Med. Chem. 54, 5592-5596 (2011).

22. Lin, L. et al. A novel small molecule, LLL12, inhibits STAT3 phosphorylation and activities and exhibits potent growth-suppressive activity in human cancer cells. Neoplasia 12, 39-50 (2010).

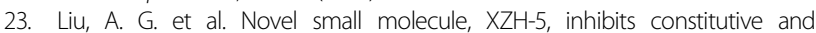
interleukin-6-induced STAT3 phosphorylation in human rhabdomyosarcoma cells. Cancer Sci. 102, 1381-1387 (2011).

24. Page, B. D. et al. Inhibiting aberrant signal transducer and activator of transcription protein activation with tetrapodal, small molecule Src homology 2 domain binders: promising agents against multiple myeloma. J. Med. Chern. 56, 7190-7200 (2013).

25. Li, S. S. et al. Discovery of oral-available resveratrol-caffeic acid based nybrids inhibiting acetylated and phosphorylated STAT3 protein. Eur. J. WTE the 124, 1006-1018 (2016).

26. Yu, W. Y., Xiao, H., Lin, J. \& Li, C. L. Discovery of novel STAT mall molec inhibitors via in silico site-directed fragment-based drug esigi Med. Chem. 56, 4402-4412 (2013).

27. Zhang, W. D. et al. Antagonizing STAT3 activatior with benzo[b]thio, shene 1, 1-dioxide based small molecules. Eur. J. Med. CF m. 125, 538-550 (2017).

28. Zhang, $X$. et al. A novel inhibitor of STAT3 hodimeriz tion selectively suppresses STAT3 activity and malignant trans tion Cancer Res. 73, 1922-1933 (2013).

29. Zhang, $X$. et al. A novel small-mole un phosphotyrosine interactions and STAT3 $d$ cpengent tumor processes. Biochem. Pharmacol. 79, 1398-140, 010).

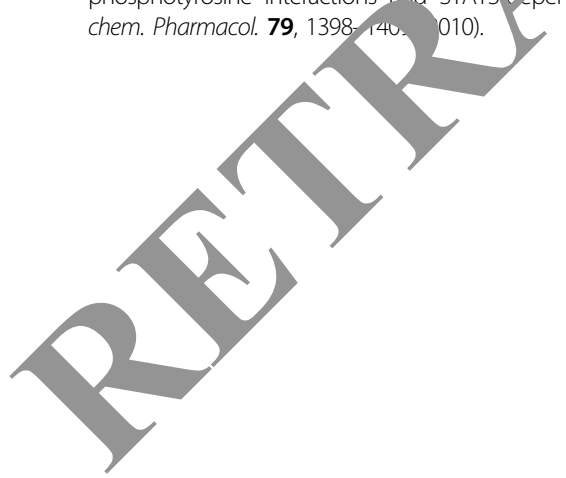

30. Zhang, X. et al. Orally bioavailable small-molecule inhibitor of transcription factor STAT3 regresses human breast and lung cancer xenografts. Proc. Natl. Acad. Sci. USA 109, 9623-9628 (2012).

31. Huang, W. et al. Small-molecule inhibitors targeting the DNA-binding domain of STAT3 suppress tumor growth, metastasis and STAT3 target gene expression in vivo. Oncogene 35, 783-792 (2015).

32. Becker, S., Groner, B. \& Muller, C. W. Three-dimensional structure of the STAT3beta homodimer bound to DNA. Nature 394, 145-151 (199\%).

33. Kotha, A. et al. Resveratrol inhibits Src and STAT3 signaling an indo'res the apoptosis of malignant cells containing activated STAT3 protels al cance Ther. 5, 621-629 (2006).

34. Johnson, D. E. et al. Targeting the IL-6/JAK/STAT3 sign'/ing axis in can Nat. Rev. Clin. Oncol. 15, 234-248 (2018).

35. Hughes, K.\& Watson, C. J. The multifaceted role STA mam rary gland involution and breast cancer. Int. J. Mol. Sci. 9, 1695 (2018,

36. Chen, Y. et al. Anti-AIDS agents 86. Synthe and anti-HNY evaluation of 2',3'seco-3'-nor DCP and DCK analogues. Eu Med. Crem. 46, 4924-4936 (2011).

37. Chen, Y. et al. Antitumor agent 92 . study of s- and o-substit 7-merca hydroxy-coumarins and chromones as potent yto agents. Lur. J. Med. Chem. 49, 74-85 (2012).

38. Yang, $X$. et al. Anti agents 20 design, synthesis, SAR, and biological studies of nove netero om-incorporated antofine and cryptopleurine analogues as poten ititumor agents. J. Med. Chem. 54, 5097-5107 (2011).

39. Cragg, ( 1 \& Newmà .J. Nature: a vital source of leads for anticancer drug developr unem. Rev. 8, 313-331 (2009).

40. Shin, D. S. et al. 9 , ptotanshinone inhibits constitutive signal transducer and activator ol transcription 3 function through blocking the dimerization in DU145 pros, ate cancer cells. Cancer Res. 69, 193-202 (2009).

41. V., Saud, S. M., Young, M. R., Colburn, N. H. \& Hua, B. Cryptotanshinone, a 3 inhibitor, suppresses colorectal cancers' proliferation and growth in itro. Mol. Cell. Biochem. 406, 63-73 (2015).

42. Larly Breast Cancer Trialists' Collaborative Group (EBCTCG). Effects of chemotherapy and hormonal therapy for early breast cancer on recurrence and 15-year survival: an overview of the randomised trials. Lancet 365, 1687-1717 (2005).

43. Hughes, K., Wickenden, J. A., Allen, J. E. \& Watson, C. J. Conditional deletion of Stat3 in mammary epithelium impairs the acute phase response and modulates immune cell numbers during post-lactational regression. J. Pathol. 227, 106-117 (2012)

44. Liu, Y. et al. Dithiaarsanes induce oxidative stress-mediated apoptosis in HL-60 cells by selectively targeting thioredoxin reductase. J. Med. Chem. 57, 5203-5211 (2014).

45. Zhou, T. et al. Design, synthesis, and evaluation of 1R,2R-dicamphanoyl-3,3dimethyldihy-dro-pyrano-[2,3-c]xanth-en-7(1H)-one (DCX) derivatives as novel anti-HIV agents. Eur. J. Med. Chem. 47, 86-96 (2012). 